\title{
The association of DAT gene methylation with striatal DAT availability in healthy subjects
}

Kyoungjune Pak ${ }^{1 *+}$, J Ju Won Seok ${ }^{2 \dagger}$, Hyun-Yeol Nam ${ }^{3 \dagger}$, Seongho Seo ${ }^{4}$, Myung Jun Lee ${ }^{5}$, Keunyoung Kim ${ }^{1}$ and In Joo Kim ${ }^{1}$

\begin{abstract}
Background: DNA methylation inhibits gene expression by preventing transcription factors from binding to DNA. Functioning of nigrostriatal dopaminergic neurons is influenced by the expression of the dopamine transporter (DAT), and genetic variations in the gene encoding DAT contribute to differences in reward processing. We aimed to investigate the action of DAT methylation on DAT protein expression measured by positron emission tomography (PET).

Methods: The emission data were acquired over 90 min with 50 frames after injection of ${ }^{18} \mathrm{~F}$-FP-CIT using PET. Binding potentials $\left(\mathrm{BP}_{\mathrm{ND}} \mathrm{s}\right)$ of ventral striatum, caudate nucleus, putamen were measured with the simplified reference tissue method. Genomic DNA was extracted from subjects' blood sampling. Methylation of 4 regions in SLC6A3 gene was assessed using bisulfite pyrosequencing. The mean percentage of methylation (\%) for each cluster was calculated by taking the average of all $\mathrm{CpG}$ site methylation levels measured within the cluster. Subjects were assessed with the Generalized Reward and Punishment Expectancy Scales (GRAPES) that consists of 30 items related with the reward and punishment that individuals expect for their behaviors.

Results: Thirty-five healthy males, with an age range between 20 and 30 years, and a mean age of $24.4 \pm 2.7$ years, were included in this study. The mean percentage of methylation (\%) from cluster $C$ showed a trend of positive correlation with DAT availability of ventral striatum ( $r$ o $=0.3712, p=0.0281$ ), not significant after correction for multiple comparisons, and a significant correlation with GRAPES A: reward expectancy scale (rho $=0.7178, p<0.0001)$.

Conclusion: DAT methylation from peripheral blood showed a trend of positive correlation with DAT availability of ventral striatum in healthy subjects; however, it was not significant after correction for multiple comparison. The degrees of methylation from cluster $C$ of DAT in peripheral blood were significantly correlated with reward scales of GRAPES A: reward expectancy scale. The association between DAT methylation and DAT expression needs to be investigated further.
\end{abstract}

Keyword: Dopamine plasma membrane transport proteins, Methylation, Neuroimaging

*Correspondence: ilikechopin@me.com

${ }^{\dagger}$ Kyoungjune Pak, Ju Won Seok, and Hyun-Yeol Nam contributed equally to this work

${ }^{1}$ Department of Nuclear Medicine and Biomedical Research Institute, Pusan National University Hospital, 179 Gudeok-ro, Seo-gu, Busan 49241, Republic of Korea

Full list of author information is available at the end of the article

\section{Background}

The dopamine transporter (DAT) is a sodium chloridedependent transmembrane protein on the presynaptic dopaminergic nerve terminal [1], regulating levels of dopamine by reuptake [2, 3]. As DAT binding is correlated with the density of dopaminergic neurons [4], decreased DAT uptake represents dopaminergic neurodegeneration such as PD [4], a neurodegenerative disease with death of dopaminergic neurons in the substantia nigra pars compacta $[5,6]$. The most sensitive imaging 
techniques for the diagnosis of neurodegeneration are positron emission tomography (PET) and single photon emission computed tomography (SPECT) by using ligands that report nigrostriatal dopaminergic function [3], which allow quantifying dopaminergic system in human brain [7].

Epigenetic change is a heritable phenotype change without alterations in the DNA sequence, such as DNA methylation. DNA methylation is an epigenetic process that adds methyl groups to cytosines of the genome, mainly in CpG sites [8]. As DNA methylation inhibits gene expression by preventing transcription factors from binding to DNA [9], DNA methylation is inversely associated with protein expression [10]. Functioning of nigrostriatal dopaminergic neurons is influenced by DAT, and genetic variations in the gene encoding DAT contribute to differences in reward processing $[11,12]$. However, in a previous study by Wiers et al., striatal DAT availability measured by ${ }^{11} \mathrm{C}$-cocaine PET did not show any significant association with DAT methylation of peripheral blood in healthy subjects [13], which is the only report that investigated the association between them. Therefore, we aimed to investigate the action of DAT methylation on DAT protein expression measured by PET and reward scales measured with the Generalized Reward and Punishment Expectancy Scales (GRAPES) [14].

\section{Methods}

\section{PET scan}

Thirty-five healthy, male subjects without brain injury, neuropsychological disorders were included in this study. The majority of the participants in this study were included in a previous study [15]. An intravenous bolus injection of ${ }^{18} \mathrm{~F}$-FP-CIT was administered. The emission data were acquired over $90 \mathrm{~min}$ with 50 frames of progressively increasing durations $(15 \mathrm{~s} \times 8$ frames, $30 \mathrm{~s} \times 16$ frames, $60 \mathrm{~s} \times 10$ frames, $240 \mathrm{~s} \times 10$ frames, and $300 \mathrm{~s} \times 6$ frames) using the Siemens Biograph 40 Truepoint PET/CT (Siemens Healthcare, Knoxville, Tennessee, USA). The dynamic PET data were collected in the 3-dimensional mode, with 148 slices with image sizes of $256 \times 256$ and pixel sizes of $1.3364 \times 1.3364 \mathrm{~mm}^{2}$. These were reconstructed using iterative method with a Gaussian filter.

\section{Image analysis}

For a volume-of-interest-based analysis, an averaged image (0-10 minafterinjection) was created from dynamic PET frames and spatially normalized to ${ }^{15} \mathrm{O}$-Water PET template in statistical parametric mapping 5 (Wellcome Trust Centre for Neuroimaging, United Kingdom). To extract time-activity curves (TACs) of volume of interest from full dynamic PET scans, Oxford-GSK-Imanova striatal atlas (https://fsl. fmrib.ox.ac.uk/fsl) for ventral striatum, caudate nucleus, putamen were applied. DAT availability, expressed in terms of binding potential $\left(\mathrm{BP}_{\mathrm{ND}}\right)$, was measured by analyzing TACs with the simplified reference tissue method [16] with the cerebellum as a reference using pmod version 3.6 (PMOD Technologies LLC, Zurich, Switzerland).

\section{DNA extraction and methylation analysis}

Genomic DNA was extracted from subjects' blood sampling. Methylation of 4 regions in SLC6A3 gene was assessed using bisulfite pyrosequencing. After bisulfite treatment of the DNA (EpiTect Fast DNA Bisulfite Kit, Qiagen, Hilden Germany), polymerase chain reaction test was done with DNA Engine Tetrad 2 Peltier Thermal Cycler (Bio-Rad, CA, USA). Primer Design was designed with PyroMark Assay Design 2.0 (Qiagen, Hilden Germany). Pyrosequencing was done with PyroMark Q48 Autoprep System (Qiagen, Hilden Germany). PyroMark Q48 Autoprep 2.4.2 Software (Qiagen, Hilden Germany) was used to assess methylation of each CpG site, 4 regions in SLC6A3 gene. The percentage of methylated alleles was divided by the total number of alleles (methylated and unmethylated) to determine the level of methylation at each $\mathrm{CpG}$ site. The mean percentage of methylation (\%) for each cluster was calculated by taking the average of all $\mathrm{CpG}$ site methylation levels measured within the cluster.

\section{Questionnaire}

Subjects were assessed with GRAPES [14]. GRAPES consists of 30 items related with the reward and punishment that individuals expect for their behaviors, GRAPES (A) reward expectancy, GRAPES (B) punishment expectancy.

\section{Statistical analysis}

Normality was assessed using the D'Agostino \& Pearson normality test. Spearman correlation test was done to determine the association of DAT methylation with $\mathrm{BP}_{\mathrm{ND}} \mathrm{S}$ and GRAPES. As 4 clusters of DAT methylation were included in this study, significance was set at $a=0.05 / 4(0.0125)$ to correct for multiple comparisons. All analyses were conducted using Prism (v7.0d, GraphPad Software Inc, La Jolla, CA, USA).

\section{Results}

Thirty-five healthy males, with an age range between 20 and 30 years, and a mean age of $24.4 \pm 2.7$ years, were included in this study. Coordinates and the percentage of methylated alleles (\%) for all SLC6A3 CpG sites are presented in Table 1 . The mean percentages of methylation (\%) were 9.9 (cluster A), 76.1 (cluster B), 91.6 (cluster C), $75.1 \%$ (cluster D), respectively. The mean 
Table 1 Coordinates and the percentage of methylated alleles (\%) for all SLC6A3 CpG sites

\begin{tabular}{|c|c|c|c|c|c|c|c|}
\hline Assay ID & CpG ID & Cluster & From ATG & From TSS & GRCh37/hg19 & Region & $\begin{array}{l}\text { The percentage of } \\
\text { methylated alleles } \\
(\%)\end{array}$ \\
\hline ADS2165RS2 & 237 & A & -3177 & -944 & 1446489 & Promoter & 13.43 \\
\hline ADS2165RS2 & 236 & A & -3174 & -941 & 1446486 & Promoter & 16.98 \\
\hline ADS2165RS2 & 235 & A & -3167 & -934 & 1446479 & Promoter & 9.30 \\
\hline ADS2165RS2 & 234 & A & -3163 & -930 & 1446475 & Promoter & 10.15 \\
\hline ADS2165RS2 & 233 & A & -3151 & -918 & 1446463 & Promoter & 6.03 \\
\hline ADS2165RS2 & 232 & A & -3148 & -915 & 1446460 & Promoter & 9.79 \\
\hline ADS2165RS1 & 231 & A & -3134 & -901 & 1446446 & Promoter & 8.85 \\
\hline ADS2165RS1 & 230 & A & -3132 & -899 & 1446444 & Promoter & 4.38 \\
\hline ADS2127FS & 547 & B & 26,817 & 29,050 & 1416496 & Intron 6 & 79.69 \\
\hline ADS2127FS & 548 & B & 26,856 & 29,089 & 1416504 & Intron 6 & 72.46 \\
\hline ADS2126FS2 & 551 & C & 27,008 & 29,241 & 1416305 & Exon 7 , Intron 6 & 95.82 \\
\hline ADS2126FS2 & 552 & C & 27,010 & 29,243 & 1416303 & Exon 7 , Intron 6 & 88.41 \\
\hline ADS2126FS & 553 & C & 27,038 & 29,271 & 1416275 & Exon 7, Intron 6 & 98.75 \\
\hline ADS2126FS & 554 & C & 27,047 & 29,280 & 1416266 & Exon 7, Intron 6 & 83.28 \\
\hline ADS2796FS1 & 1149 & $\mathrm{D}$ & 48,635 & 50,868 & 1394678 & Exon $153^{\prime}$ UTR & 70.80 \\
\hline ADS2796FS1 & 1150 & $\mathrm{D}$ & 48,640 & 50,873 & 1394673 & Exon $153^{\prime}$ UTR & 96.21 \\
\hline ADS2796FS1 & 1151 & $\mathrm{D}$ & 48,646 & 50,879 & 1394667 & Exon 15 3'UTR & 65.53 \\
\hline ADS2796FS1 & 1152 & D & 48,653 & 50,886 & 1394660 & Exon $153^{\prime}$ UTR & 78.86 \\
\hline ADS2796FS1 & 1153 & $\mathrm{D}$ & 48,656 & 50,889 & 1394657 & Exon $153^{\prime}$ UTR & 62.88 \\
\hline ADS2796FS2 & 1154 & $\mathrm{D}$ & 48,679 & 50,912 & 1394634 & Exon $153^{\prime}$ UTR & 69.95 \\
\hline ADS2796FS2 & 1155 & $\mathrm{D}$ & 48,682 & 50,915 & 1394631 & Exon $153^{\prime}$ UTR & 69.51 \\
\hline ADS2796FS2 & 1156 & $\mathrm{D}$ & 48,697 & 50,930 & 1394616 & Exon $153^{\prime}$ UTR & 92.09 \\
\hline ADS2796FS2 & 1157 & $\mathrm{D}$ & 48,709 & 50,942 & 1394604 & Exon $153^{\prime} U T R$ & 70.38 \\
\hline
\end{tabular}

percentage of methylation (\%) from each cluster did not show any association each other (Fig. 1). The mean percentage of methylation (\%) from each cluster was not associated with DAT availability of the ventral striatum, showing a trend of positive correlation between that from cluster $\mathrm{C}$ and DAT availability of the ventral striatum without correction ( $r h o=0.3712, p=0.0281$ ), which was not significant after correction for multiple comparison. In addition, the mean percentage of methylation (\%) from cluster $C$ was positively correlated with
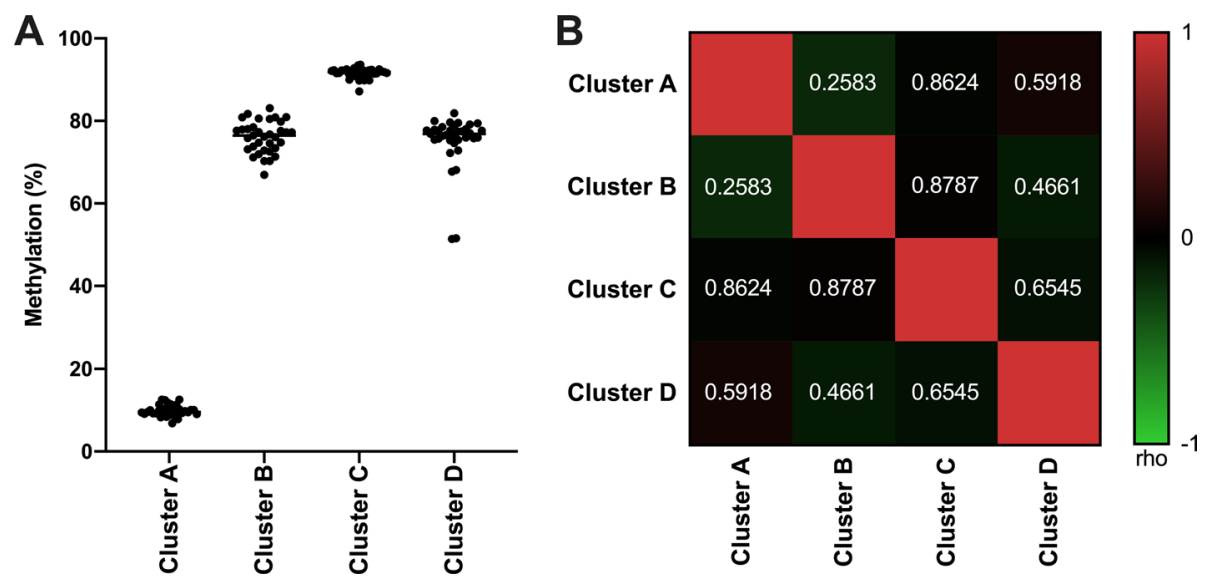

Fig. 1 a Distribution of mean percentage of methylation (\%) for each cluster and $\mathbf{b}$ inter-correlation of mean percentage of methylation (\%) 

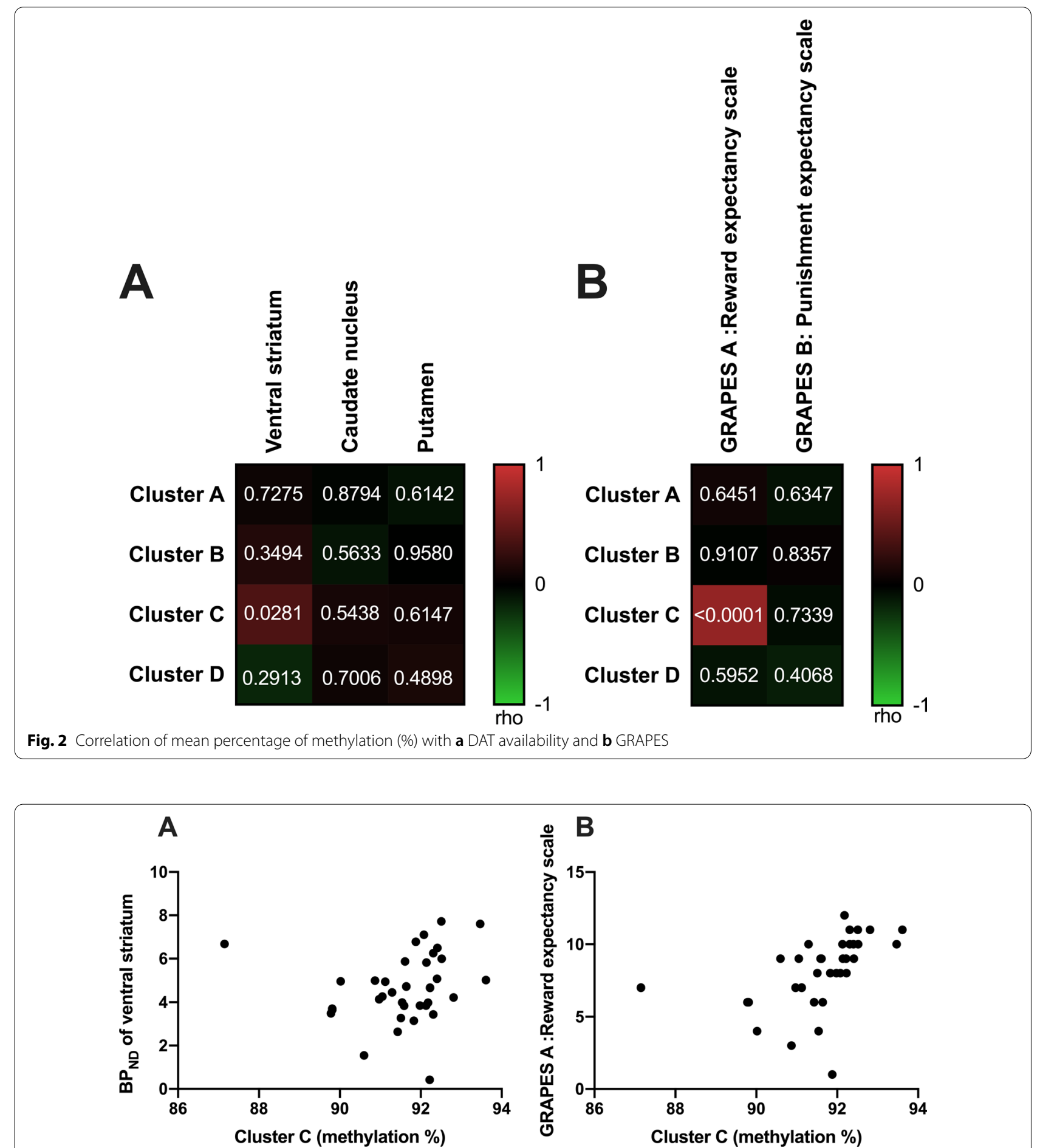

Fig. 3 Correlation of mean percentage of methylation (\%) from cluster $C$ with a DAT availability and $\mathbf{b}$ GRAPES 
GRAPES A: reward expectancy scale $(\mathrm{rho}=0.7178$, $p<0.0001$ ) (Figs. 2, 3).

\section{Discussion}

In this study, the degrees of methylation from cluster $\mathrm{C}$ (Exon 7) of DAT in peripheral blood showed a trend of positive correlation with DAT availability of the ventral striatum and were significantly correlated with reward scales of GRAPES A: reward expectancy scale.

This is the first study that investigated the association between the degree of methylation of DAT and DAT availability of PET in healthy subjects. Previously, the association of methylation of DAT gene with DAT availability was investigated in patients with attention-deficit hyperactivity disorder (ADHD) [13], which is the only study of DAT methylation and PET. Although methylation of DAT gene was not significantly different between patients with ADHD and healthy subjects, DAT availability measured by ${ }^{11} \mathrm{C}$-cocaine PET was negatively correlated with the degree of DAT methylation in patients with ADHD, not in healthy subjects [13]. This correlation was significant in both caudate nucleus, putamen, and ventral striatum [13]. However, the underlying mechanisms of insignificancy between DAT availability and DAT methylation in healthy subjects remained unclear. In subjects with alcohol dependency, the effect of DAT methylation was investigated with reward processing [10] and depressive symptoms [17] using functional magnetic resonance imaging. DAT methylation in promoter region predicted activation of nucleus accumbens during the anticipation of high and low loss in healthy subjects, not in subjects with alcohol dependency [10]. In addition, subjects with alcohol dependency showed that activity of amygdala to alcohol cue was correlated with DAT methylation only those with low depression scores [17]. Also, higher DAT methylation predicted the relapse of alcohol dependency [17].

Epigenetic change is a heritable phenotype change without alterations in the DNA sequence, such as DNA methylation. DNA methylation is a process that adds methyl groups to cytosines of the genome, mostly in CpG sites [8]. DNA methylation inhibits gene expression by preventing transcription factors from binding to DNA [9]. Therefore, DNA methylation has shown to be inversely associated with protein expression [10]. In rodent, DAT methylation was negatively associated with striatal DAT expression [18]. In humans, only one study reported the association between DAT methylation and DAT availability measured by ${ }^{11} \mathrm{C}$-cocaine PET as explained above [13]. Inconsistent with the study by Wiers et al., we demonstrated a trend of positive association between DAT methylation and DAT availability measured by ${ }^{18} \mathrm{~F}$-FP-CIT PET. In addition, the mean percentage of methylation (\%) from cluster $\mathrm{C}$ was strongly correlated with GRAPES A: reward expectancy scale, similar to the study by Muench et al., which predicted striatal responses during reward processing by DAT methylation in healthy subjects, not in alcoholdependent individuals [10]. DAT methylation in alcoholdependent individuals [10], or ADHD [13], showed the difference with that in healthy subjects. Therefore, DAT methylation might have an implication on treatment of alcohol dependency or ADHD.

There are several points that should be considered in these results. First, it is unclear that whether DAT methylation obtained from peripheral blood has an association with DAT methylation of brain. In this regard, Wiers et al. proved the significant correlation between DAT methylation of substantia nigra and DAT methylation from peripheral blood both in healthy subjects and patients with ADHD, however, showing moderate correlation in both promotor region $(r=0.44)$, and CpG site $230(r=0.53)$ of healthy subjects [13]. Second, the mean percentage of methylation was calculated from each cluster and considered as the degree of DAT methylation level. However, the degree of DAT methylation may be different within each cluster, and the region of each cluster may be different. Third, the radiopharmaceuticals used in measurement of DAT availability were different in each study. Theoretically, as DNA methylation inhibits or regresses the binding of transcription factors [9], DNA methylation might be negatively associated with protein expression [10]. However, at least with regard to DAT, this association is unclear until now as we showed a trend of positive association between DAT methylation and DAT availability, and Wiers et al. showed no significant association [13]. The mechanisms underlying these findings might be complex and likely vary across genes. Also, as a small number of subjects were included in this study, further studies of a larger number of subjects with a broader age range should be carried out in the future.

\section{Conclusion}

DAT methylation from peripheral blood showed a trend of positive correlation with DAT availability of ventral striatum in healthy subjects; however, it was not significant after correction for multiple comparison. The degrees of methylation from cluster C of DAT in peripheral blood were significantly correlated with reward scales of GRAPES A: reward expectancy scale. The association between DAT methylation and DAT expression needs to be investigated further.

\section{Authors' contributions}

Kyoungjune Pak took part in study design and writing the manuscript. Ju Won Seok, Hyun-Yeol Nam, Seongho Seo involved in image analysis. Myung Jun 
Lee participated in image analysis and study design. Keunyoung Kim took part in image analysis. In Joo Kim involved in writing the manuscript. All authors read and approved the final manuscript.

\section{Funding}

This research was supported by Basic Science Research Program through the National Research Foundation of Korea (2020R1F1A1054201).

\section{Availability of data and materials}

All data are available to corresponding author of the manuscript upon reasonable request.

\section{Declarations}

\section{Ethics approval and consent to participate}

All procedures performed in studies involving human participants were in accordance with the ethical standards of the institutional and/or national research committee and with the 1964 Declaration of Helsinki and its later amendments or comparable ethical standards. Ethical permission for the study procedures was obtained from the Institutional Review Boards at Pusan National University Hospital. Subject consent has been obtained by Pusan National University Hospital. This article does not contain any studies with animals performed by any of the authors.

\section{Consent for publication}

Not applicable.

\section{Competing interests}

The authors declare that they have no competing interests.

\section{Author details}

'Department of Nuclear Medicine and Biomedical Research Institute, Pusan National University Hospital, 179 Gudeok-ro, Seo-gu, Busan 49241, Republic of Korea. ${ }^{2}$ Department of Nuclear Medicine, Chung-Ang University College of Medicine, Seoul, Republic of Korea. ${ }^{3}$ Department of Nuclear Medicine, Samsung Changwon Hospital, Sungkyunkwan University School of Medicine, Changwon, Republic of Korea. ${ }^{4}$ Department of Electronic Engineering, Pai Chai University, Daejeon, Republic of Korea. ${ }^{5}$ Department of Neurology, Pusan National University Hospital, Busan, Republic of Korea.

Received: 31 March 2021 Accepted: 9 June 2021

Published online: 12 June 2021

\section{References}

1. Vaughan RA, Foster JD. Mechanisms of dopamine transporter regulation in normal and disease states. Trends Pharmacol Sci. 2013;34(9):489-96.

2. Marshall V, Grosset D. Role of dopamine transporter imaging in routine clinical practice. Mov Disord. 2003;18(12):1415-23.

3. Booth TC, Nathan M, Waldman AD, Quigley AM, Schapira AH, Buscombe $J$. The role of functional dopamine-transporter SPECT imaging in parkinsonian syndromes, part 1. AJNR Am J Neuroradiol. 2015;36(2):229-35.

4. Park E. A new era of clinical dopamine transporter imaging using 123I-FPCIT. J Nucl Med Technol. 2012;40(4):222-8.

5. Nalls MA, Pankratz N, Lill CM, Do CB, Hernandez DG, Saad M, et al. Largescale meta-analysis of genome-wide association data identifies six new risk loci for Parkinson's disease. Nat Genet. 2014;46(9):989-93.

6. Rawlik K, Rowlatt A, Tenesa A. Imputation of DNA methylation levels in the brain implicates a risk factor for Parkinson's disease. Genetics. 2016;204(2):771-81.

7. Zipursky RB, Meyer JH, Verhoeff NP. PET and SPECT imaging in psychiatric disorders. Can J Psychiatry. 2007;52(3):146-57.

8. Jin B, Li Y, Robertson KD. DNA methylation: superior or subordinate in the epigenetic hierarchy? Genes Cancer. 2011;2(6):607-17.

9. Robertson KD. DNA methylation and human disease. Nat Rev Genet. 2005:6(8):597-610.

10. Muench C, Wiers CE, Cortes CR, Momenan R, Lohoff FW. Dopamine transporter gene methylation is associated with nucleus accumbens activation during reward processing in healthy but not alcohol-dependent individuals. Alcohol Clin Exp Res. 2018:42(1):21-31.

11. Dreher JC, Kohn P, Kolachana B, Weinberger DR, Berman KF. Variation in dopamine genes influences responsivity of the human reward system. Proc Natl Acad Sci USA. 2009;106(2):617-22.

12. Hahn T, Heinzel S, Dresler T, Plichta MM, Renner TJ, Markulin F, et al. Association between reward-related activation in the ventral striatum and trait reward sensitivity is moderated by dopamine transporter genotype. Hum Brain Mapp. 2011;32(10):1557-65.

13. Wiers CE, Lohoff FW, Lee J, Muench C, Freeman C, Zehra A, et al. Methylation of the dopamine transporter gene in blood is associated with striatal dopamine transporter availability in ADHD: a preliminary study. Eur J Neurosci. 2018;48(3):1884-95.

14. Samuel A, Ball MZ. Sensation seeking, Eysenck's personality dimensions and reinforcement sensitivity in concept formation. Pers Individ Differ. 1990;11(4):343-53.

15. Pak K, Seo S, Kim K, Lee MJ, Shin MJ, Suh S et al. Striatal dopamine transporter changes after glucose loading in humans. Diabetes Obes Metab. 2020;22(1):116-122

16. Lammertsma AA, Hume SP. Simplified reference tissue model for PET receptor studies. Neuroimage. 1996;4(3 Pt 1):153-8.

17. Wiers CE, Shumay E, Volkow ND, Frieling H, Kotsiari A, Lindenmeyer J, et al Effects of depressive symptoms and peripheral DAT methylation on neural reactivity to alcohol cues in alcoholism. Transl Psychiatry. 2015;5:e648.

18. Kim P, Choi CS, Park JH, Joo SH, Kim SY, Ko HM, et al. Chronic exposure to ethanol of male mice before mating produces attention deficit hyperactivity disorder-like phenotype along with epigenetic dysregulation of dopamine transporter expression in mouse offspring. J Neurosci Res. 2014;92(5):658-70.

\section{Publisher's Note}

Springer Nature remains neutral with regard to jurisdictional claims in published maps and institutional affiliations.

\section{Submit your manuscript to a SpringerOpen ${ }^{\circ}$ journal and benefit from:}

- Convenient online submission

- Rigorous peer review

- Open access: articles freely available online

- High visibility within the field

Retaining the copyright to your article

Submit your next manuscript at springeropen.com 